\title{
Challenges and Management of Long COVID in Individuals with Hematological Illnesses
}

\author{
Dana Yelin ${ }^{a, b} \quad$ Ili Margalit ${ }^{a, b, c}$ \\ ${ }^{a}$ COVID Recovery Clinic, Rabin Medical Center, Beilinson Hospital, Petah Tikva, Israel; ${ }^{b}$ Faculty of Medicine, Tel Aviv \\ University, Tel Aviv, Israel; 'Infectious Diseases Unit, Rabin Medical Center, Beilinson Hospital, Petah Tikva, Israel
}

\section{Keywords \\ COVID-19 · Long COVID · Rehabilitation · Persistent symptoms}

\begin{abstract}
COVID-19 has impacted hundreds of millions of people globally, a relatively large proportion of whom continue to suffer from ongoing, sometime debilitating symptoms. This phenomenon, termed "long COVID," is difficult to diagnose and manage because of a paucity of objective findings and despite the abundance of descriptive data published so far. In this review, we aimed to describe the common manifestations of long COVID, diagnostic and management challenges, and address specific aspects in hematologic patients.
\end{abstract}

(c) 2022 S. Karger AG, Basel

\section{Introduction}

COVID-19 has impacted hundreds of millions of people globally [1]. Initially, authorities and healthcare systems have mainly focused on the acute phase of the disease because of its immediate life-threatening complications and mortality. This approach has underestimated the impact of COVID-19 on survivors, a relatively large proportion of whom continue to suffer from ongoing, sometime debilitating symptoms.

Post-COVID-19, also known as "Long COVID," a term first coined by the suffering patients themselves, is a phenomenon characterized by persistent symptoms following recovery from COVID-19 [2]. The World Health Organization defines "post COVID-19 condition" as " $a$ history of probable or confirmed SARS-CoV-2 infection, usually 3 months from the onset of COVID-19 with symptoms that last for at least 2 months and cannot be explained by an alternative diagnosis." [2] In this review, we will refer to this condition as long COVID.

The most common long COVID symptoms are weakness, fatigue, dyspnea, cognitive impairment, and smell and taste disturbances (Table 1) [3-5]. This is reminiscent of previously reported fatigue syndromes following other infectious diseases, such as Epstein-Barr virus, dengue virus, chikungunya, and $\mathrm{Q}$ fever or other syndromes with unknown pathophysiology such as chronic fatigue syndrome and fibromyalgia $[6,7]$.

It is imperative to discern between long COVID and specific postinfectious syndromes occurring following COVID-19. The latter relates to several distinct entities with previously established pathophysiology that associ- 
Table 1. Typical long COVID symptoms (over 12 weeks from infection) by prevalence, data adopted from systematic review, Michelen et al. [3]

\begin{tabular}{|c|c|c|c|}
\hline Symptom & Proportion $(95 \% \mathrm{Cl})$ & $\begin{array}{l}\text { Number of studies } \\
\text { reporting }\end{array}$ & $\begin{array}{l}\text { Total number of } \\
\text { included individuals }\end{array}$ \\
\hline Weakness & $41.2(25.4-59.0)$ & 2 & 513 \\
\hline Fatigue & $31.0(23.9-39.0)$ & 17 & 6,039 \\
\hline Breathlessness/exertional dyspnea & $25.1(17.9-34.0)$ & 20 & 5,523 \\
\hline Sweat/night sweats & $23.7(20.7-27.1)$ & 2 & 683 \\
\hline Weight loss* & $21.0(8.1-44.5)$ & 2 & 568 \\
\hline Loss of appetite & $17.5(4.1-51.0)$ & 3 & 1,906 \\
\hline Smell disturbance & $15.2(10.8-21.0)$ & 19 & 5,668 \\
\hline Hair loss & $14.3(5.3-33.2)$ & 5 & 2,810 \\
\hline Taste disturbances & $13.5(9.0-19.9)$ & 17 & 5,423 \\
\hline Myalgia & $11.3(6.2-19.8)$ & 12 & 4,782 \\
\hline Palpitations & $9.7(6.0-15.3)$ & 8 & 4,778 \\
\hline Arthralgia & $9.4(5.7-15.0)$ & 9 & 3,960 \\
\hline Paresthesia & $9.1(2.2-30.9)$ & 2 & 257 \\
\hline Cough & $8.2(4.9-13.4)$ & 16 & 5,031 \\
\hline Nausea and vomiting & $6.7(1.6-23.6)$ & 4 & 821 \\
\hline Chest pain & $6.4(3.2-12.4)$ & 11 & 4,878 \\
\hline Nasal congestion & $5.0(2.7-8.9)$ & 3 & 1,003 \\
\hline Headache & $4.9(2.3-10.1)$ & 11 & 4,535 \\
\hline Vision disturbances & $4.8(3.3-6.8)$ & 2 & 586 \\
\hline Flushing & $4.8(3.2-7.0)$ & 1 & 538 \\
\hline Sore throat & $4.7(2.4-8.9)$ & 5 & 2,896 \\
\hline Dizziness & $4.5(2.5-7.9)$ & 5 & 3,141 \\
\hline Walking/gait abnormality & $4.2(2.0-8.5)$ & 3 & 809 \\
\hline Diarrhea & $4.0(2.1-7.6)$ & 10 & 3,925 \\
\hline Tremors & $3.5(0.3-30.6)$ & 3 & 1,124 \\
\hline Skin rash & $2.8(1.0-8.2)$ & 4 & 2,374 \\
\hline Abdominal pain & $2.3(0.5-9.4)$ & 4 & 1,427 \\
\hline Fever & $1.1(0.2-4.7)$ & 7 & 3,624 \\
\hline \multicolumn{4}{|c|}{ Psychosocial and neurocognitive symptoms } \\
\hline Reduced quality of life & $36.8(18.4-60.0)$ & 3 & 807 \\
\hline Concentration impairment & $26.0(21.0-31.7)$ & 2 & 254 \\
\hline Anxiety & $18.7(8.9-35.3)$ & 7 & 3,551 \\
\hline Sleep disorders & $18.2(9.6-31.6)$ & 9 & 3,442 \\
\hline Memory impairment & $17.9(5.3-46.3)$ & 5 & 886 \\
\hline PTSD & $9.1(3.7-21.0)$ & 6 & 2,057 \\
\hline Depression & $8.1(4.1-15.1)$ & 6 & 3,662 \\
\hline Confusion & $2.7(1.9-3.8)$ & 2 & 1,218 \\
\hline
\end{tabular}

$\mathrm{Cl}$, confidence interval; PTSD, post-traumatic stress disorder. * Most studies do not report weight loss as a long COVID symptom. The very few studies that did consider it among the typical symptoms mostly included individuals recovered from severe disease and ICU survivors.

ate with COVID-19 and tend to appear during the postinfectious period, usually up to 12 weeks from diagnosis. These include peri-myocarditis, thyroiditis, idiopathic thrombocytopenic purpura, Guillain-Barré syndrome [8-12]. There is a scientific basis implying that these may appear following infectious diseases, and their association with COVID is plausible, albeit necessitating additional proof through population-based studies.
There are conflicting reports on the prevalence of long COVID, ranging from $5 \%$ and up to $\sim 80 \%$ depending on the population surveyed, classification definitions, severity of the acute illness, and the time elapsed from the acute episode [3, $4,13,14]$. The prevalence may be subjected to change over time, reflecting the emergence of new viral variants (e.g., delta and omicron variants) and the effect of vaccines on disease severity and potentially on long COVID itself [15]. 
The risk factors for long COVID established thus far include a positive association with the severity of the acute illness, and higher risk among women $[4,16,17]$. Middle-aged individuals (40-60 years old) are also at an increased risk of long COVID in comparison to younger adults $[18,19]$; however, this may be confounded by disease severity and warrants further research on the subject $[20,21]$.

\section{Long COVID in Individuals Diagnosed with Hematological Diseases}

There are currently no publications reporting on the prevalence of long COVID among individuals diagnosed with hematological diseases. It is reasonable to assume that the prevalence of long COVID in this particular subpopulation is at least as high as reported for the general population. Moreover, individuals with hematological diseases are at higher risk for COVID-19 infection and its complications and are less likely to develop an adequate immune response to COVID-19 vaccines. Consequently, it is likely that their proportion among individuals with long COVID is higher than their realistic portion in the general population.

Long COVID symptoms are mostly nonspecific and multisystemic by nature. Accordingly, they share many similarities with symptoms that are suggestive of the underlying hematological disease or side effects of treatment. This is particularly relevant as fatigue and other typical long COVID symptoms are not uncommon post intensive antineoplastic therapy. The only exception is anosmia which is particularly suggestive of long COVID.

Long-term symptoms following COVID-19 in hematological patients may challenge clinical decision-making and may lead to unnecessary worries and redundant diagnostic procedures and complications; on the other hand, individuals with systemic manifestations arising from recurrence of their underlying hematological illness or opportunistic infections may be mistakenly addressed as having long COVID, delaying their diagnosis and hindering necessary treatment.

\section{Hematological Syndromes Post-COVID-19}

Acute COVID-19 is associated with hematological complications including venous thromboembolism, disseminated intravascular coagulation, thrombocytopenia, anemia, and hemophagocytic lymphohistiocytosis [22].

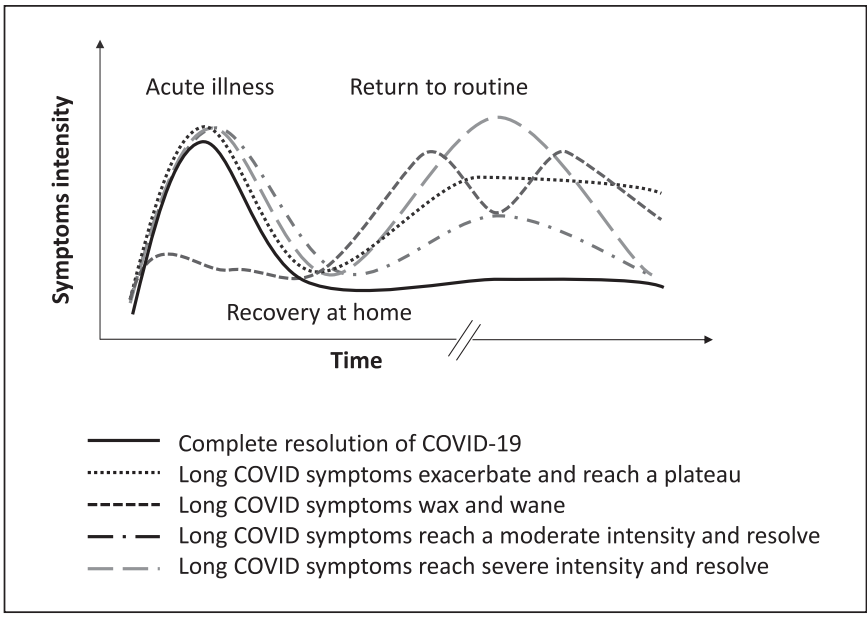

Fig. 1. Typical courses of symptom intensity over time in individuals with long COVID.

Some of these were also reported following recovery. These include idiopathic thrombocytopenic purpura [11, 23], autoimmune hemolytic anemia [10], Evans syndrome [11], and hemophagocytic lymphohistiocytosis [24]. Further population-based studies are required in order to assert whether these associations, if exists at all, are causal or merely circumstantial. Similar to other acute illnesses, ongoing risk for post recovery venous thromboembolism is a concern $[25,26]$. This topic, which justifies an in-depth consideration, is beyond the scope of the current review.

\section{Long COVID Symptoms}

Long COVID is characterized by an abundance of somatic and psychosocial symptoms, while the physical examination as well as imaging and blood tests are usually unremarkable. The most frequently reported symptoms are weakness, fatigue, dyspnea, cognitive impairment, and smell and taste disturbances (Table 1) [3, 4]. These symptoms vary in their intensity and can become debilitating, impairing one's ability to regain pre-illness occupational and social functioning. The course of symptoms may also vary between individuals. While most report improvement over time, some report constant symptoms, a waxing and waning pattern or even a worsening trend (Fig. 1). The duration of long COVID is variable, and many of the afflicted individuals continue to suffer for long time periods [27, 28], and reports of symptoms lasting for 12 months and more are abundant $[16,18,29,30]$. 
Several pathophysiological mechanisms have been hypothesized, primarily, immune dysregulation, and endotheliopathy affecting the lungs, heart, and central or peripheral nervous systems. However, these hypotheses have not been established and are currently under investigation $[31,32]$. Considering that longitudinal studies reported a substantial decrease in the prevalence of each of the long COVID symptoms, it is reasonable to assert that long COVID is a self-limiting medical condition. However, considering its burden, debilitating nature, and the fact that a non-negligible group of individuals may suffer for extremely long periods, effort must be taken in order to assess interventions aiming at shortening the time period for complete symptoms resolution.

\section{Diagnosis of Long COVID}

In the absence of specific signs or other leading diagnostic clues, long COVID is diagnosed by the exclusion of other clinical conditions. The evaluation of individuals with ongoing symptoms following COVID-19 relies on meticulous history taking and physical examination. Whenever these imply an established postinfectious entity (see above), the workup should be directed at the suspected entity. In the appropriate context, after excluding signs suggesting an alternative diagnosis, in a patient who reports typical symptoms (Table 1), long COVID can be confidently assumed.

It should be emphasized that long COVID is usually not accompanied by abnormalities in vital signs, physical examination or blood parameters. Clinicians should therefore consider such abnormalities as alarming signs, suggesting another diagnosis. These signs include prolonged fever, night sweats, unexplained weight loss, significantly elevated markers of inflammation, and any other abnormal laboratory test. Although weight loss has been reported as a long COVID symptom in 2 studies [3], we do not consider it among the typical long COVID symptoms. Therefore, a thorough investigation is advised in these patients.

\section{Distinguishing between Long and Persistent/ Relapsed COVID}

Persistent COVID is a discrete and relatively rare entity, recognized in individuals with immune suppression, mostly reported in patients with lymphoproliferative disorders and/or treatment with anti-CD-20 monoclonal antibodies [33-35]. Although it shares clinical similari- ties with long COVID in terms of ongoing symptoms, it involves ongoing viral replication and elevated inflammatory markers and reflects a failure of the immune system to achieve virological clearance. Relapsed COVID-19, a more controversial entity, relates to a persistent infection, in which there was documentation of virological clearance ( 2 consecutive negative polymerase chain reaction tests between the positive results) [36].

Distinguishing persistent COVID from long COVID in immunosuppressed individuals may be challenging, as nasopharyngeal polymerase chain reaction may be positive intermittently for a long duration even in immunocompetent individuals [36]. Ongoing fever, elevated inflammatory markers, or other alarming signs (see above) continuing into the expected recovery phase in an immunosuppressed individual are the hallmark of persistent COVID and should not be confused with long COVID.

\section{Management}

\section{Acknowledging the Clinical Syndrome and Patient} Reassurance

In addition to the suffering arising from the debilitating symptoms, many individuals who experience long COVID withstand doubt, shame, and social stigma. One of the most important roles of physicians caring for individuals with long COVID is to acknowledge their patients' suffering. Many of the sufferers find comfort in the discovery that their harsh experience is a common phenomenon. Physicians are therefore encouraged to normalize the experience of their patients by giving it a name and to diagnose it as long COVID.

In the absence of evidence-based interventions, it is particularly important to reassure the patient. We advise physicians to share with their patients the data that has been gathered so far. It is important to convey the message that long COVID generally carries a good prognosis and tends to resolve over time. We also advise physicians to inform their patients that the duration of symptoms might be very long and that it is currently impossible to forecast the individual course of symptoms.

\section{Psychosocial Evaluation and Intervention}

Many of the individuals with long COVID experience anxiety and emotional distress (Table 1). These manifestations likely intensify other somatic and cognitive long COVID symptoms. Initial assessment should include screening for emotional distress and further referral to social workers and/or psychotherapists when necessary. 
Whenever appropriate, physicians and psychotherapists can conduct the clinical visits together. This holistic approach of multidisciplinary care is beneficent.

\section{Physical and Pulmonary Rehabilitation}

Patients following severe or critical disease tend to suffer significant deconditioning and most require rehabilitation, be it in-hospital or at an outpatient facility. Postdischarge rehabilitation has been shown to be beneficial for survivors of severe or critical COVID-19 [37-39]. However, decreased exercise capacity also occurs in individuals with long COVID following mild or moderate acute disease. Most of these do not require additional evaluation, but patients with severe dyspnea, dyspnea at rest, chest pain on exercise and any abnormal physical finding on examination may need referral for additional studies such as pulmonary function testing, echocardiography, and cardiopulmonary exercise testing [40, 41].

No comparative interventional studies have been published thus far for managing long COVID in the mediumlong term. We recommend that all patients be encouraged to gradually resume or take up low-intensity physical activity such as walking or swimming. Patients with a sedentary lifestyle prior to COVID-19 should try to begin a regular albeit very light exercise program (even a few minutes at a time) and increase gradually [42]. Patients with prior active lifestyle should be advised avoiding an abrupt return to their previous exercise schedule. Gradual increase of exercise intensity and duration should be adjusted to patients' ability and postexercise recovery rate. Recommendations for professional athletes have been proposed by several authors $[42,43]$. Even in the absence of evidence in mild-moderate recoverees, it is plausible to offer physiotherapy or hydrotherapy to patients unable to return to their occupational or recreational pre-illness routine.

\section{Cognitive Assessment and Rehabilitation}

Long COVID cognitive symptoms usually include impaired executive functions such as working memory, concentration, and attention [3, 44]. This may severely impair the quality of life and daily functioning of the sufferers. At the extreme of the spectrum, working individuals sometimes find themselves unable to implement their occupational duties and maintain their employment. Retired or elderly individuals may encounter decline in their quality of life, as they feel unable to conduct recreational or leisure activities as was prior to COVID-19.

For individuals with mild cognitive symptoms and no overt influence on their quality of life, we recommend re-

Long COVID in Individuals with

Hematological Illnesses turning to premorbid occupational and recreational routine as early as possible. Daily reading, solving crossword puzzles, Sudoku, or any other cognition challenging activities are also recommended. It is advised to reserve assessment by occupational therapist and neurologist for those with severe functional impairment or diminished quality of life. Cognitive rehabilitation by a trained occupational therapist could provide coping strategies to limit impact on daily life and may accelerate the rate of improvement [45].

\section{Olfactory Training}

Individuals recovering from COVID-19 often experience anosmia, dysgeusia, parosmia, and phantosmia. Many of them describe these symptoms as devastating, impairing their quality of life. For long COVID symptoms involving changes in taste and smell, we suggest olfactory training. This protocol was developed for cases of postinfectious olfactory dysfunction and was proven effective in several randomized control trials $[46,47]$ prior to COVID-19. A single randomized control trial has examined use of nasal corticosteroids with olfactory training versus training alone and has shown both interventions as equally effective in long COVID patients, with no added benefit for the corticosteroid group [48]. Considering that olfactory training carries no potential harms or side effects (as opposed to corticosteroids), it is recommended for long COVID, whereas it is advised to avoid addition of corticosteroids [47].

\section{Summary}

Long COVID is a debilitating entity that affects the life of millions of sufferers worldwide. Since its pathophysiology is yet to be understood, interventions aimed at improving quality of life and hasten symptoms' resolution rate are still lacking. Physicians caring for these patients should focus their efforts on three main aspects: rule out other entities; patient reassurance; and referral for rehabilitation whenever appropriate.

Individuals with hematological illnesses, most notably those with malignancies, constitute a particular subpopulation in several ways. They are more susceptible to COVID-19 and possibly to long COVID as well. Moreover, their nonspecific and systemic long COVID symptoms mimic disease progression or recurrence and therefore may pose diagnostic dilemmas. Hematologists should be aware of this phenomenon and be familiar with the typical symptoms as well as alarming signs implying other diagnoses. This approach will spare patients unnecessary distress and diagnostic procedures. Furthermore, a holis- 
tic approach, reassurance, and rehabilitation programs may provide these individuals with comfort and hope, merits that potentially have therapeutic value, which is of particular need at times of no proven directed treatments.

\section{Conflict of Interest Statement}

The authors report no conflict of interest.

\section{Funding Sources}

No funding was received for this study.

\section{Author Contributions}

D.Y. and I.M. have contributed equally to the conceptualization and writing of this article.

\section{References}

1 WHO. WHO Coronavirus disease (COVID-19) dashboard (live) [Internet] [cited 2021 Feb 27]. Available from: https://covid19. who.int/.

2 World Health Organization. A clinical case definition of post COVID-19 condition by a Delphi consensus. 2021 Oct. Available from: https: //www.who.int/publications/i/item/ WHO-2019-nCoV-Post_COVID-19_condition-Clinical_case_definition-20211.

3 Michelen M, Manoharan L, Elkheir N, Cheng V, Dagens A, Hastie C, et al. Characterising long COVID: a living systematic review. BMJ Glob Heal. 2021;6(9):1-12.

4 Sudre CH, Murray B, Varsavsky T, Graham MS, Penfold RS, Bowyer RC, et al. Attributes and predictors of long COVID. Nat Med. 2021;27(4):626-31.

5 Yelin D, Margalit I, Yahav D, Runold M, Bruchfeld J. Long COVID-19: it's not over until? Clin Microbiol Infect. 2021;27(4):5068.

6 Hickie I, Davenport T, Wakefield D, VollmerConna U, Cameron B, Vernon SD, et al. Postinfective and chronic fatigue syndromes precipitated by viral and non-viral pathogens: prospective cohort study. Br Med J. 2006; 333(7568):575

7 Lam MHB, Wing YK, Yu MWM, Leung CM, Ma RCW, Kong APS, et al. Mental morbidities and chronic fatigue in severe acute respiratory syndrome survivors long-term followup. Arch Intern Med. 2009;169(22):2142-7.

8 Scoppettuolo P, Borrelli S, Naeije G. Neurological involvement in SARS-CoV-2 infection: a clinical systematic review. Brain Behav Immun Heal. 2020;5:100094

9 Sanghvi SK, Schwarzman LS, Nazir NT. Cardiac MRI and myocardial injury in COVID-19: diagnosis, risk stratification and prognosis. Diagnostics. 2021;11(1):130.

10 Pelle MC, Tassone B, Ricchio M, Mazzitelli M, Davoli C, Procopio G, et al. Late-onset myocardial infarction and autoimmune haemolytic anaemia in a COVID-19 patient without respiratory symptoms, concomitant with a paradoxical increase in inflammatory markers: a case report. J Med Case Rep. 2020;14(1): 246.

11 Bhattacharjee S, Banerjee M. Immune thrombocytopenia secondary to COVID-19: a sys- tematic review. SN Compr Clin Med. 2020; 14(11):2048-9.

12 Marazuela M, Giustina A, Puig-Domingo M. Endocrine and metabolic aspects of the COVID-19 pandemic. Rev Endocr Metab Disord. 2020;21(4):495-507.

13 Nehme M, Braillard O, Chappuis F, Courvoisier DS, Guessous I. Prevalence of symptoms more than seven months after diagnosis of symptomatic COVID-19 in an outpatient setting. Ann Intern Med. 2021;174(9):125260.

14 Huang C, Huang L, Wang Y, Li X, Ren L, Gu $\mathrm{X}$, et al. Articles 6-month consequences of COVID-19 in patients discharged from hospital: a cohort study. Lancet. 2021;10270(9): 220-32.

15 Antonelli M, Penfold RS, Merino J, Sudre CH, Molteni E, Berry S, et al. Risk factors and disease profile of post-vaccination SARS-CoV-2 infection in UK users of the COVID symptom study app: a prospective, community-based, nested, case-control study. Lancet Infect Dis. 2022;22(1):43-55.

16 Zhang X, Wang F, Shen Y, Zhang X, Cen Y, Wang B, et al. Symptoms and health outcomes among survivors of COVID-19 infection 1 year after discharge from hospitals in Wuhan, China. JAMA Netw Open. 2021 Sep; 4(9):e2127403.

17 Bai F, Tomasoni D, Falcinella C, Barbanotti $\mathrm{D}$, Castoldi R, Mulè G, et al. Female gender is associated with "long COVID" syndrome: a prospective cohort study. Clin Microbiol Infect. 2021;S1198-743X(21)00629-7.

18 Boscolo-Rizzo P, Guida F, Polesel J, Marcuzzo AV, Capriotti V, D'Alessandro A, et al. Sequelae in adults at 12 months after mild-tomoderate coronavirus disease 2019 (COVID-19). Int Forum Allergy Rhinol. 2021; 11(12):1685-8.

19 Lombardo MDM, Foppiani A, Peretti GM, Mangiavini L, Battezzati A, Bertoli S, et al. Long term COVID-19 complications in inpatients and outpatients: a One-Year Follow Up Cohort Study. SSRN Electron J. 2021;8:1-8.

20 Tenforde MW, Kim SS, Lindsell CJ, Billig Rose E, Shapiro NI, Files DC, et al. Symptom duration and risk factors for delayed return to usual health among outpatients with COVID-19 in a multistate health care systems network: United States, March-June 2020. MMWR Morb Mortal Wkly Rep. 2020;69: 993-8.

21 Munblit D, Bobkova P, Spiridonova E, Shikhaleva A, Gamirova A, Blyuss O, et al. Incidence and risk factors for persistent symptoms in adults previously hospitalized for COVID-19. Clin Exp Allergy. 2021;51(9): 1107-20.

22 Terpos E, Ntanasis-Stathopoulos I, Elalamy I, Kastritis E, Sergentanis TN, Politou M, et al. Hematological findings and complications of COVID-19. Am J Hematol. 2020;95(7):83447.

23 Kuter DJ. Exacerbation of immune thrombocytopenia following COVID-19 vaccination. Br J Haematol. 2021 Nov; 195(3):365-70.

24 Serrano C, Español I, Cascales A, Moraleda JM, Kalita P, Laishram D, et al. Secondary hemophagocytic lymphohistiocytosis in postCOVID-19 patients: a report of two cases. SN Compr Clin Med. 2021;13(12):2389-92.

25 Pretorius E, Vlok M, Venter C, Bezuidenhout JA, Laubscher GJ, Steenkamp J, et al. Persistent clotting protein pathology in long $\mathrm{CO}$ VID/post-acute sequelae of COVID-19 (PASC) is accompanied by increased levels of antiplasmin. Cardiovasc Diabetol. 2021; 20(1):1-18.

26 Zuin M, Rigatelli G, Zuliani G, Roncon L. The risk of thrombosis after acute-COVID-19 infection. QJM An Int J Med. 2021 Sep;114(9): 619-20.

27 Havervall S, Rosell A, Phillipson M, Mangsbo SM, Nilsson P, Hober S, et al. Symptoms and functional impairment assessed 8 months after mild COVID-19 among health care workers. Jama. 2021;11:11-3.

28 Peghin M, Palese A, Venturini M, De Martino $\mathrm{M}$, Gerussi V, Graziano E, et al. Post-COVID-19 symptoms 6 months after acute infection among hospitalized and non-hospitalized patients. Clin Microbiol Infect. 2021 Jun; S1198-743X(21)00281-0.

29 Munblit D, Bobkova P, Spiridonova E, Shikhaleva A, Gamirova A, Blyuss O, et al. Risk factors for long-term consequences of $\mathrm{CO}$ VID-19 in hospitalised adults in Moscow using the ISARIC Global follow-up protocol: StopCOVID cohort study. medRxiv. 2021; 2021. 02.17.21251895. 
30 Huang L, Yao Q, Gu X, Wang Q, Ren L, Wang $\mathrm{Y}$, et al. 1-year outcomes in hospital survivors with COVID-19: a longitudinal cohort study. Lancet. 2021;398(10302):747-58.

31 Crook H, Raza S, Nowell J, Young M, Edison P. Long covid: mechanisms, risk factors, and management. BMJ. 2021;374:1-18.

32 Fogarty H, Townsend L, Morrin H, Ahmad A, Comerford C, Karampini E, et al. Persistent endotheliopathy in the pathogenesis of long COVID syndrome. J Thromb Haemost. 2021 Oct;19(10):2546-53.

33 Reuken PA, Stallmach A, Pletz MW, Brandt C, Andreas N, Hahnfeld S, et al. Severe clinical relapse in an immunocompromised host with persistent SARS-CoV-2 infection. Leukemia. 2021;35(3):920-3.

34 Helleberg M, Niemann CU, Moestrup KS, Kirk O, Lebech A-M, Lane C, et al. Persistent COVID-19 in an immunocompromised patient temporarily responsive to two courses of remdesivir therapy. J Infect Dis. 2020 Sep; 222(7):1103-7.

35 Truong TT, Ryutov A, Pandey U, Yee R, Goldberg L, Bhojwani D, et al. Persistent SARS-CoV-2 infection and increasing viral variants in children and young adults with impaired humoral immunity. medRxiv Prepr Serv Heal Sci. 2021 Mar;2021.02.27.21252099.

36 Yahav D, Yelin D, Eckerle I, Eberhardt CS, Wang J, Cao B, et al. Definitions for coronavirus disease 2019 reinfection, relapse and PCR re-positivity. Clin Microbiol Infect. 2020;27:315-8.
37 Liu K, Zhang W, Yang Y, Zhang J, Li Y, Chen Y. Respiratory rehabilitation in elderly patients with COVID-19: a randomized controlled study. Complement Ther Clin Pract. 2020;39:101166.

38 Gloeckl R, Leitl D, Jarosch I, Schneeberger T, Nell C, Stenzel N, et al. Benefits of pulmonary rehabilitation in COVID-19: a prospective observational cohort study. ERJ Open Res. 2021;7(2):00108-2021.

39 Goodwin VA, Allan L, Bethel A, Cowley A, Cross JL, Day J, et al. Rehabilitation to enable recovery from COVID-19: a rapid systematic review. Physiotherapy. 2021 Jun;111:4-22.

40 Jennings G, Monaghan A, Xue F, Mockler D, Romero-Ortuño R. A systematic review of persistent symptoms and residual abnormal functioning following acute COVID-19: ongoing symptomatic phase vs. post-COVID-19 syndrome. medRxiv. 2021;10(24):5913.

41 Cassar MP, Tunnicliffe EM, Petousi N, Lewandowski AJ, Xie C, Mahmod M, et al. Symptom persistence despite improvement in cardiopulmonary health: insights from longitudinal CMR, CPET and lung function testing post-COVID-19. EClinicalMedicine. 2021;41:101159.

42 Halle M, Bloch W, Niess AM, Predel H, Reinsberger C, Scharhag J, et al. Exercise and sports after COVID 19: guidance from a clinical perspective. Transl Sport Med. 2021;4(3): 310-8.
43 Małek ŁA, Marczak M, Miłosz-Wieczorek B, Konopka M, Braksator W, Drygas W, et al. Cardiac involvement in consecutive elite athletes recovered from Covid-19: a magnetic resonance study. J Magn Reson Imaging. 2021;53(6):1723-9.

44 Becker JH, Lin JJ, Doernberg M, Stone K, Navis $\mathrm{A}$, Festa JR, et al. Assessment of cognitive function in patients after COVID-19 infection. JAMA Netw Open. 2021;4(10):8-11.

45 Watters K, Marks TS, Edwards DF, Skidmore ER, Giles GM. A framework for addressing clients' functional cognitive deficits after COVID-19. Am J Occup Ther. 2021 Jul-Aug; 75(Suppl 1):7511347010p1.

46 Webster KE, O’Byrne L, MacKeith S, Philpott C, Hopkins C, Burton MJ. Interventions for the prevention of persistent post-COVID-19 olfactory dysfunction. Cochrane database Syst Rev. 2021;7:CD013877.

47 Addison AB, Wong B, Ahmed T, Macchi A, Konstantinidis I, Huart C, et al. Clinical Olfactory Working Group consensus statement on the treatment of postinfectious olfactory dysfunction. J Allergy Clin Immunol. 2021; 147(5):1704-19.

48 Abdelalim AA, Mohamady AA, Elsayed RA, Elawady MA, Ghallab AF. Corticosteroid nasal spray for recovery of smell sensation in COVID-19 patients: a randomized controlled trial. Am J Otolaryngol. 2021 Mar-Apr;42(2): 102884 . 